\title{
Comparación entre la posición de Valdivia y la posición prona en la nefrolitectomía percutánea (NLP)
}

\author{
Amón Sesmero JH, Del Valle González N, Conde Redondo C, Rodriguez Toves A, \\ Cepeda Delgado M, Martínez-Sagarra Oceja JM.
}

Servicio de Urología. Hospital Rio Hortega. Valladolid.

Actas Urol Esp. 2008;32(4):424-429

\begin{abstract}
RESUMEN
COMPARACIÓN ENTRE LA POSICIÓN DE VALDIVIA Y LA POSICIÓN PRONA EN LA NEFROLITECTOMIIA PERCUTÁNEA (NLP)

Introducción: La posición supina, ideada por Valdivia hace dos décadas para la nefrolitectomía percutánea (NLP), a pesar de aventajar a la posición prona en aspectos concernientes a la anestesia y a la ergonomía quirúrgica, no ha tenido una amplia difusión en la comunidad urológica debido a que se ha asociado a una mayor dificultad técnica, menor tasa de limpieza de cálculos y mayor tasa de complicaciones, aunque los escasos estudios comparativos existentes no sustenten estos argumentos. En este trabajo se comparan ambas posiciones en la NLP analizando los resultados desde el punto de vista de la dificultad técnica, la eficacia en la resolución de la litiasis y de las complicaciones.

Material y Métodos: Una serie de 50 pacientes a los que se les realizó una NLP en posición supina fue comparada de forma retrospectiva con otra de 54 pacientes intervenidos consecutivamente por NLP en posición prona. Todas las intervenciones fueron realizadas bajo anestesia general, predominando los abordajes del cáliz inferior y los trayectos únicos sobre los múltiples en ambos grupos. La dilatación del tracto de nefrostomía se realizó mayoritariamente con catéter balón de alta presión. El área litiásica media tratada fue de $399,93 \pm 58,2 \mathrm{~mm}^{2}$ en el grupo supino y de $416,36 \pm 46,54 \mathrm{~mm}^{2}$ para el prono, $(\mathrm{p}=0,456)$. La manipulación de la litiasis se llevó a cabo mediante fragmentación ultrasónica, mecánica y en un reducido número de pacientes se realizó extracción directa con pinzas.

Resultados: Ambos grupos fueron semejantes en cuanto a los parámetros demográficos y a variables referentes a la técnica quirúrgica como el número de trayectos realizados, cáliz elegido para la punción, tipo de dilatación del tracto de nefrostomía o clase de energía utilizada en la fragmentación. Se fracasó en el acceso a las cavidades renales en 3 casos en cada grupo (6\% para el grupo supino y 5,56\% para el prono, (p=0,716)). El tiempo operatorio medio fue de $74,55 \pm 25,54 \mathrm{~min}$. para el grupo supino frente a $91,82 \pm 24,82 \mathrm{~min}$. para el prono, (p=0,123). En el postoperatorio inmediato se observó, mediante una radiografía simple, una tasa de limpieza de cálculo de $76 \%$ para el grupo en supino y del $74 \%$ para el grupo en prono, ( $\mathrm{p}=0,308$ ) Se empleó LEOCH como tratamiento complementario en el $12 \%$ de los pacientes del grupo supino y en el 12,96\% del prono, (p=0,478), y se realizó una segunda NLP por litiasis residual en $4(8 \%)$ y en 3 pacientes $(5,56 \%)$ del grupo supino y del prono respectivamente $(\mathrm{p}=0,697)$.

Hubo equivalencia en el postoperatorio de ambos grupos en cuanto a los días de hospitalización $(5,89 \pm 4,7$ para el supino $v s 5,5 \pm 4,09$ para el prono, $\mathrm{p}=0,694)$, y en cuanto a la analgesia que precisaron $(6,89 \pm 4,87$ dosis en el supino frente a $6,18 \pm 4,09$ dosis en el prono, ( $\mathrm{p}=0,580$ ). No hubo diferencias entre la tasa de complicaciones, que fue baja para ambos grupos, si bien, en un caso del grupo supino se produjo una lesión del colon.

Conclusión: La posición de Valdivia es igual de factible que la posición prona en la NLP. Las tasas, tanto de éxito en cuanto a resolución litiásica, como de complicaciones son similares entre ambas posiciones.

Palabras clave: Posición supina. Posición de Valdivia. Nefrolitectomía percutánea.
\end{abstract}

\section{ABSTRACT}

COMPARISON BETWEEN VALDIVIA POSITION AND PRONE POSITION IN PERCUTANEOUS NEPHROLITHOTOMY

Introduction: Although the supine position created by Dr. Valdivia two decades ago to perform the procedure known as percutaneous nephrolitectomy (PNL) presents advantages against the prone position in some aspects concerning anesthesia and surgical ergonomy, its use has failed to spread widely among the urology community due to certain technical difficulties, a lower rate of calculi clearing and a higher rate of complications, in spite of the fact that the scarce comparative studies do not show enough data to support this opinion. The present study compares both positions considering the technical difficulties encountered, their effectiveness and their results and complications.

Material and Methods: A series of 50 patients that underwent PCNL by prone position is compared retrospectively with another series of 54 patients that underwent consecutively PCNL by prone position. All procedures were performed under general anesthesia, the inferior calyx approach was the one used the most over the supracostal approach, and the sole tract over the multi-tract approach was predominant. Dilatation of the nephrostomy tract was done, in most of the cases, with a high-pressure balloon catheter. The stone surface treated was $399.93 \pm 58.2$ mm ${ }^{2}$ for the supine group, and $416.36 \pm 46.54 \mathrm{~mm}^{2}$ for the prone one $(\mathrm{p}=0.456)$. The management of the stones was carried out by ultrasonic or ballistic fragmentation, and a small group of patients underwent direct stone removal.

Results: As far as demographic parameters and operative variables such as number of tracts performed, calyx election, type of tract dilatation and kind of energy used for fragmentation, both groups were homogeneous. In 3 cases of each group there was a failure to access the kidney. The rate of failure was $6 \%$, and $5.56 \%$, for the supine and prone groups, respectively $(\mathrm{p}=0.716)$. Average operating time was $74.55 \pm 25.54$ and $91.82 \pm$ 24.82 minutes, respectively, $\mathrm{p}=0.123$. A postoperative $\mathrm{x}$-ray showed a stone-free rate of $76 \%$ for the supine group and $74 \%$ for the prone group, $\mathrm{p}=0.308$. ESWL was the supplementary treatment for $12 \%$ of the patients in the supine group, and for $12.96 \%$ of the patients in the prone group $\mathrm{p}=$ 0.478 , and a second procedure was performed on $4(8 \%)$ patients in the supine group and on $3(5.56 \%)$ in the prone one, $\mathrm{p}=0.697$. Hospital stay was the same for both groups $(5.89 \pm 4.7$ for the supine group, and $5.5 \pm 4.09$ for the prone one, $\mathrm{p}=0.694)$. As far as analgesia required, $6.89 \pm 4.87$ was administered for the supine against $6.18 \pm 4.09$ for the prone, $\mathrm{p}=0.580$. The complications rate was very low for both groups and also very similar; one of the patients in the supine group suffered a lesion to the colon.

Conclusion: Valdivia position is as feasible as the prone position for PCNL. Success rates, as far as stone clearing, and complications are similar for both positions.

Keywords: Supine position. Valdivia position. Percutaneous nephrolitectomy. 
$\mathrm{T}_{\mathrm{h}}$ radicionalmente la cirugía percutánea renal se ha realizado en posición de decúbito prono por similitud con el acceso renal que se utilizaba para la nefrostomía temporal. El conocimiento de la anatomía topográfica del riñón en esta postura y la ligera flexión de la columna lumbar creada al apoyar el abdomen sobre una almohada, que aumenta la distancia entre la última costilla y la cresta iliaca, facilitaban la punción de las cavidades renales. Por el contrario, las alteraciones de la fisiología de la ventilación pulmonar y del retorno venoso, que se producen en esta posición como consecuencia del incremento de la presión intraabdominal, son inconvenientes importantes para la anestesia en pacientes con patología respiratoria, cardiaca u obesidad mórbida ${ }^{1-3}$.

En 1987 Valdivia introduce la posición en decúbito supino para la realización de la cirugía percutánea renal $^{4}$, aportando mejoras para el paciente desde el punto de vista de la anestesia y del tiempo quirúrgico, y para el cirujano, al realizar la cirugía en una posición más ergonómica, disminuyendo la exposición radiológica de las manos y posibilitando el acceso simultáneo de la vía excretora por vía retrógrada ${ }^{5,6}$. A pesar de estas ventajas, la posible mayor dificultad técnica y menor eficacia en cuanto a tasa de limpieza de cálculo, que se imputan a esta posición así como la resistencia por parte del urólogo a modificar una posición habitual para él, hacen que siga siendo más utilizada la posición prona en la cirugía percutánea renal, y sólo en los últimos años se viene reconociendo la utilidad de la posición supina en pacientes de alto riesgo o con obesidad mórbida $^{7,8}$.

Con la intención de valorar el grado de dificultad técnica y de eficacia de esta posición frente a la posición prona en la nefrolitectomía percutánea, realizamos un estudio comparativo retrospectivo de ambas, analizando aspectos técnicos, resultados y complicaciones.

\section{MATERIAL Y MÉTODOS}

Se revisan las historias clínicas de 50 pacientes afectos de litiasis renal intervenidos mediante nefrolitectomía percutánea en posición supina desde junio de 2004 a marzo de 2006 y las de otro grupo de 54 pacientes que fueron intervenidos consecutivamente por igual patología en posición prona desde enero 2002 a marzo de 2004. Se indicó la NLP en litiasis coraliformes, litiasis piélicas mayores de 2,5 $\mathrm{cm}$, fracasos de $\mathrm{LEOCH}$, litiasis de cáliz inferior o litiasis que producían obstrucción por estar enclavadas en la unión pieloureteral (UPU) o en el uréter proximal.

Las variables demográficas de ambos grupos en cuanto a edad, sexo, lado afecto y tipo de litiasis están expresadas en la Tabla 1. El área media de la litiasis tratada en el grupo supino fue de $399,93 \pm 58,2 \mathrm{~mm}^{2}$ y de $416,36 \pm 46,54 \mathrm{~mm}^{2}$ en el prono $(\mathrm{p}=0,456)$.

Tabla 1. Variables demográficas

\begin{tabular}{lccc}
\hline & $\begin{array}{c}\text { Posición supina } \\
\text { (n=50) }\end{array}$ & $\begin{array}{c}\text { Posición prona } \\
\text { (n=54) }\end{array}$ & valor $\boldsymbol{p}$ \\
\hline Edad media & $54,1 \pm 15,10$ & $53,9 \pm 19,49$ & 0,430 \\
Varón/hembra & $23 / 27$ & $30 / 24$ & 0,206 \\
Derecho/izquierdo & $26 / 24$ & $30 / 24$ & 0,910 \\
$\begin{array}{l}\text { Tipo litiasis: } \\
\quad \text { Piélica }\end{array}$ & 22 & & \\
$\quad \begin{array}{l}\text { Cáliz sup. } \\
\text { Caliz inf. }\end{array}$ & 3 & 24 & \\
$\quad \begin{array}{l}\text { UPU } \\
\text { Coraliforme }\end{array}$ & 14 & 6 & 0,487 \\
$\begin{array}{l}\text { Área litiasica } \\
\text { (mm2): }\end{array}$ & $399,93 \pm 58,2$ & $416,36 \pm 46,54$ & 0,456 \\
\hline
\end{tabular}

En todos los pacientes la intervención se realizó bajo anestesia general. En ambos grupos se realizó un cateterismo retrógrado previo con intención de opacificar y distender las cavidades renales para facilitar la punción renal que se realizó bajo control radiológico.

Para el acceso percutáneo en posición prona, el cateterismo se realizó en posición de litotomía y posteriormente se volteó al paciente apoyando su abdomen sobre una almohada para extender la región lumbar y disminuir la movilidad renal.

En el grupo de pacientes intervenidos en posición supina se utilizó la posición de Valdivia modificada por el grupo de Galdácano ${ }^{12}$ que permite realizar el cateterismo retrogrado y la punción sin ningún cambio postural (Fig. 1). Para ello, se apoyó el flanco afecto del paciente sobre una bolsa de suero de 3 litros, (lo que procura una lateralización del tronco de 20$25^{\circ}$ aproximadamente) y las extremidades inferiores se colocaron separadas en ligera flexión para realizar un acceso simultáneo a la vía excretora por vía retrógrada. La punción en esta posición se realizó a la altura de la línea axilar posterior (Fig. 2). 


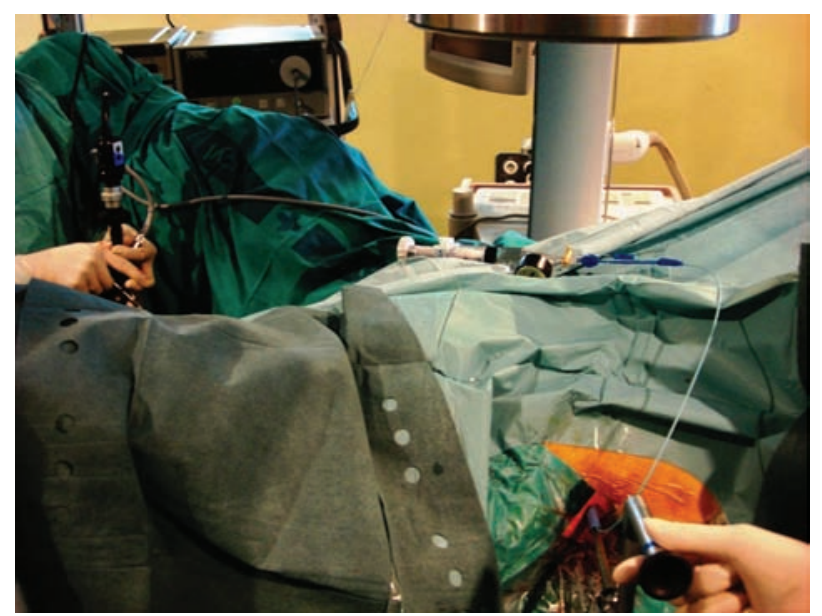

FIGURA 1. Acceso combinado. Posición de Valdivia modificada (P. de Galdácano).

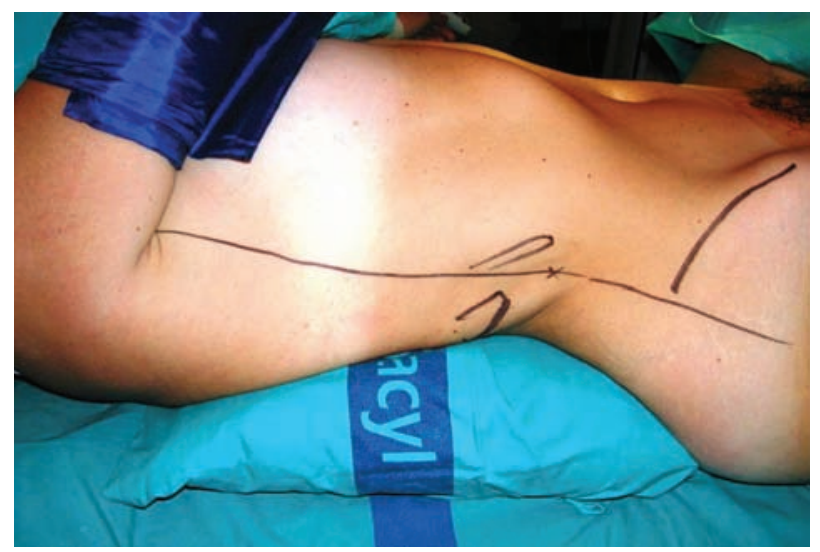

FIGURA 2. Linea axilar posterior, donde se realizará la punción.

En el 93,3\% de los pacientes la dilatación del tracto de nefrostomía se llevó a cabo con catéter balón de alta presión tipo Nefromax (Boston Scientific Corporation), excepto en un pequeño grupo (7casos), en el que se utilizaron los dilatadores metálicos de Alken.

La manipulación litiásica se llevó a cabo mediante fragmentación ultrasónica y mecánica principalmente. En un pequeño número de casos se realizó extracción directa de la litiasis con pinzas. Los porcentajes de cada grupo así como otras variables referentes a la intervención como el cáliz abordado o el número de trayectos realizados están recogidos en la Tabla 2.

Para el estudio estadístico se utilizó el paquete estadístico correspondiente, aplicándose el test de la "t" de Student para comparar una variable cuantitativa con otra cualitativa y la prueba del chi cuadrado para la comparación de dos variables cualitativas.
Tabla 2. Parámetros intervención

\begin{tabular}{|c|c|c|c|}
\hline & Pos. Supino & Pos. Prono & valor $p$ \\
\hline \multicolumn{4}{|l|}{$\mathrm{N}^{0}$ de trayectos } \\
\hline Uno & 47 & 50 & \multirow{3}{*}{0,635} \\
\hline Dos & 2 & 3 & \\
\hline Tres & 1 & 1 & \\
\hline \multicolumn{4}{|l|}{ Cáliz punción } \\
\hline Superior & 3 & 3 & \multirow{3}{*}{0,520} \\
\hline Medio & 4 & 7 & \\
\hline Inferior & 45 & 48 & \\
\hline \multicolumn{4}{|l|}{ Tipo dilatación } \\
\hline Nefromax & 48 & 49 & \multirow[t]{2}{*}{0,090} \\
\hline Alken & 2 & 5 & \\
\hline \multicolumn{4}{|l|}{ Energía litotricia } \\
\hline Ultrasónica & 15 & 18 & \multirow{4}{*}{0,921} \\
\hline Balística & 22 & 23 & \\
\hline Extracción directa & 10 & 9 & \\
\hline Nada & 3 & 4 & \\
\hline
\end{tabular}

\section{RESULTADOS}

En nuestro estudio comparativo retrospectivo no hubo diferencias con significación estadística entre los parámetros preoperatorios de ambos grupos ni tampoco en cuanto a datos referentes a la técnica como el número de trayectos realizados, cáliz elegido para la punción, tipo de dilatación del tracto de nefrostomía o clase de energía utilizada en la fragmentación, por lo que podemos considerar que los grupos comparados fueron homogéneos.

No se consiguió un acceso franco a las cavidades renales en tres casos de cada grupo, por tanto, la tasa de fracaso en la cirugía fue del 6\% para el grupo en posición supina y de 5,56 \% para el grupo prono $(p=0,716)$. El tiempo operatorio medio fue $74,55 \pm 25,54$ min. ( $r=40-180)$ en la posición supina frente a $91,82 \pm 24,82 \mathrm{~min}$. ( $\mathrm{r}=45-190)$.en la posición prona, si bien la diferencia no fue estadísticamente significativa $(\mathrm{p}=0,201)$.

En 8 pacientes del grupo supino y en 10 del grupo prono ( $16 \%$ y $18,52 \%$ respectivamente), no se dejó nefrostomía al acabar la intervención $(\mathrm{p}=0,478)$. Un drenaje interno mediante catéter doble J precisó el 20\% y el 14,8\% de los pacientes del grupo supino y del prono respectivamente $(\mathrm{p}=$ 0,116).

En el postoperatorio inmediato se observó, mediante una radiografía simple, una tasa de limpieza de cálculo de $76 \%$ para el grupo en supino y del $74 \%$ para el grupo en prono $(p=0,308)$. La litia- 
sis residual se trató mediante ESWL en 6 pacientes del grupo supino (12\%) y en 7 del grupo prono (14\%), y se indicó una segunda nefrolitectomía percutánea en 4 pacientes del grupo supino (8\%) y en 3 del grupo prono (5,56\%). En 2 pacientes del grupo supino y en otros 4 del prono la litiasis residual no fue tratada debido a su pequeño tamaño.

En el grupo supino hubo sangrado intraoperatorio que dificultó la visión endoscópica en 3 casos $(6 \%)$ y en un paciente se detectó una perforación importante de la vía excretora que obligó a detener la intervención. Dos pacientes del grupo prono (4\%) sangraron durante la intervención dificultando el procedimiento.

La tasa de transfusión postoperatoria fue de 6\% para el grupo supino y 5,56\% para el grupo prono $(p=0,343)$. En este grupo un paciente presentó hematuria persistente debida a una fístula arteriovenosa que fue tratada conservadoramente mediante embolización. Se detectó hematoma perirrenal en un paciente de cada grupo, ambos correspondieron a casos en los que no se dejó catéter de nefrostomía.
Hubo fiebre postoperatoria en el 12\% de los pacientes del grupo supino y en el $14,8 \%$ de los del grupo prono. Un paciente del grupo prono sufrió un cuadro séptico que precisó cuidados intensivos.

La media en días de ingreso hospitalario fue de $5,89 \pm 4,7$ para el grupo supino y de $5,5 \pm 4,09$ para el grupo prono $(\mathrm{p}=0,694)$. Las dosis de analgésicos recibidas en el postoperatorio fueron $6,89 \pm 4,87 \mathrm{y}$ $6,18 \pm 4,09$ para los grupos supino y prono respectivamente $(\mathrm{p}=0,580)$ (Tabla 3$)$.

\section{DISCUSIÓN}

Aunque la posición supina para la nefrolitectomía percutánea fue descrita hace casi dos décadas por Valdivia ${ }^{4}$, quién posteriormente demostró su eficacia en una importante serie de pacientes ${ }^{5}$, su empleo no se ha generalizado y solo en los últimos años parece ser más utilizada, sobre todo en pacientes de alto riesgo ${ }^{7,8,10}$.

La posición prona tiene importantes inconvenientes fisiológicos para el paciente como son la restricción en la capacidad ventilatoria ${ }^{1}$, una mayor

Tabla 3. Resultados y complicaciones

\begin{tabular}{|c|c|c|c|}
\hline & Supino & Prono & Valor $p$ \\
\hline Tasa de limpieza de litiasis & $76 \%$ & $74 \%$ & 0,308 \\
\hline Tiempo operatorio (minutos) & $74,55+25,54$ & $91,82+24,82$ & 0,123 \\
\hline Días Hospitalización & $5,89+4,7$ & $5,5+4,09$ & 0,694 \\
\hline Dosis analgesia & $6,89+4,87$ & $6,18+4,09$ & 0,580 \\
\hline Sin nefrostomía (final) & $8(16 \%)$ & $10(18,52 \%)$ & 0,478 \\
\hline Reintervención ( $\left.2^{\text {a }} \mathrm{NLP}\right)$ & $4(8 \%)$ & $3(5,56 \%)$ & 0,697 \\
\hline LEOCH complementaria & $6(12 \%)$ & $7(12,96 \%)$ & 0,478 \\
\hline Fracaso de acceso a la vía & $3(6 \%)$ & $3(5,56 \%)$ & 0,716 \\
\hline Sangrado intraoperatorio & $3(6 \%)$ & $2(4 \%)$ & \\
\hline Perforación de la vía excret. & $1(2 \%)$ & 0 & \\
\hline Imposibilidad de manipulación litiásica & $1(2 \%)$ & $2(3,7 \%)$ & \\
\hline Hematoma perirrenal & $1(2 \%)$ & $1(1,8 \%)$ & \\
\hline Transfusión & $3(6 \%)$ & $3(5,56 \%)$ & \\
\hline Fístula A-V (embolización) & 0 & $1(1,8 \%)$ & 0,423 \\
\hline Fiebre postoperatoria & $6(12 \%)$ & $8(14,8 \%)$ & \\
\hline Sepsis & 0 & $1(1,8 \%)$ & \\
\hline Fístula renocutánea & 0 & $1(1,8 \%)$ & \\
\hline Fístula cólica & $1(2 \%)$ & 0 & \\
\hline Otras (IAM) & 0 & $1(1,8 \%)$ & \\
\hline
\end{tabular}


dificultad de retorno venoso y un aumento de la presión intraocular ${ }^{3}$ que se traducen en mayor riesgo anestésico $^{2}$.

Por otro lado, la necesidad de dar la vuelta al paciente exige la concurrencia de mayor número de ayudantes en el quirófano y una vigilancia máxima en dicha maniobra para evitar complicaciones traumatológicas y neurológicas.

A pesar de que la posición supina soslaya estos inconvenientes, su difusión entre los urólogos no ha sido la esperada debido, como señalan algunos, a una presumible mayor dificultad técnica, mayor tasa de litiasis residual y mayor riesgo de perforación del colon ${ }^{11}$, si bien son muy escasos los estudios comparativos entre ambas posiciones.

En nuestro estudio no randomizado, los dos grupos se compararon respecto a las dificultades técnicas del abordaje y a la manipulación intraoperatoria de la litiasis. Respecto al primer punto, si bien observamos, durante las maniobras dilatación del tracto de nefrostomía, un desplazamiento medial del riñón mayor en el grupo supino que en la posición prona, no encontramos dificultades adicionales en el acceso al riñón. Teniendo en cuenta, además, que el acceso se realizó en la mayoría de los casos por el cáliz inferior en ambos grupos $(90 \%$ para el grupo supino y $88,8 \%$ para el prono) y que el número de trayectos múltiples y supracostales empleados fue similar, el grado de dificultad técnica fue similar en ambas posiciones, dado que nuestra tasa de fracaso en el acceso a la cavidad pielocalicial fue equivalente en ambos grupos $16 \%$ en supino y 5,56\% en prono). Una vez alcanzada la cavidad pieloureteral, en un caso del grupo supino y en 2 pacientes del grupo prono no se consiguió manipular la litiasis. Podemos concluir, pues, que ambas posiciones resultaron semejantes en cuanto a dificultad técnica.

Se ha señalado que en la posición supina el tiempo operatorio se reduce, dado que no es preciso voltear al paciente una vez se ha realizado el cateterismo retrogrado ${ }^{5}$. En nuestro trabajo, utilizando la posición supina de Valdivia modificada por el grupo de Galdácano ${ }^{12}$, el tiempo medio empleado fue 17 minutos inferior en la posición supina, y si bien esta diferencia puede explicarse por lo antes expuesto, no tuvo significación estadística, por lo que este dato no fue relevante.
La tasa de limpieza de litiasis comunicada en el metaanálisis de las series más recientes de NLP está en torno al $78 \%{ }^{13}$.En nuestro estudio la tasa de limpieza de litiasis conseguida (76\% en posición supina y $74 \%$ en posición prona) se mantiene en un rango aceptable en relación con aquélla y fue similar para los grupos comparados. Por otro lado, también la tasa de reintervención para ambos grupos de pacientes fue equivalente (se realizó una $2^{\mathrm{a}}$ NLP en 4 pacientes del grupo prono y en tres del grupo supino), por lo que podemos concluir que no hubo diferencias en cuanto a la eficacia de tratamiento entre ambas posiciones.

En cuanto a las complicaciones, debemos señalar que no hubo diferencias significativas entre ambas posiciones en cuanto a las complicaciones acaecidas tanto durante la intervención como en el postoperatorio inmediato en su análisis global. La tasa de transfusión fue similar en ambas posiciones $(6 \%$ y $5,56 \%$ para el grupo supino y prono respectivamente), y dicho porcentaje estuvo en concordancia con la comunicada en otras series de NLP en posición supina que varía del 3\% al 9\%5,8,11 $\mathrm{y}$, por otra parte, fue sensiblemente inferior a la tasa que señala el metaanálisis de la AUA para las series de NLP en posición prona ${ }^{13}$, lo que en parte atribuimos a nuestra preferencia en el empleo del balón de alta presión como sistema de dilatación del tracto de nefrostomía.

Debemos reseñar una complicación importante e infrecuente como es perforación de colon que ocurrió en la serie supina. Creemos que hasta la fecha es la primera complicación de este tipo comunicada por NLP en posición de Valdivia. Este autor y otros $5,8,9,12$ han sugerido que en esta posición el riesgo de lesión cólica disminuye debido a que se produce un desplazamiento del colon hacia la línea media. Nuestro caso correspondió a un abordaje del cáliz inferior del riñón izquierdo en una mujer delgada. Aunque la punción siempre la realizamos en la línea axilar posterior, alejada en teoría del intestino grueso, pensamos, al igual que señalan otros ${ }^{14}$, que la marcada delgadez de la paciente fue un factor de riesgo determinante en esta complicación.

Finalmente, debemos señalar que el postoperatorio fue similar para ambos grupos en cuanto a estancia hospitalaria y dosis de analgésicos que precisaron. 


\section{CONCLUSIONES}

La posición supina es tan efectiva y segura como la posición prona en la nefrolitectomía percutánea desde el punto de vista de los resultados y de las complicaciones observadas en este estudio. Por otro lado, el grado de dificultad técnica de esta cirugía en ambas posiciones es similar.

Indudablemente, ventajas como la disminución del riesgo anestésico, mayor ergonomía de trabajo para el cirujano, posibilidad de acceso retrógrado simultáneo de la vía excretora determinan que sea la posición preferible para la realización de la NLP.

\section{REFERENCIAS}

1. Peces-Barba G, Rodriguez-Nieto MJ, Verbanck S, Paiva M, González-Mangado N. Lower pulmonary diffusing capacity in the prone vs. supine posture. J Appl Physiol. 2004; 96(5):19371942.

2. Martin JT. The ventral decubitus(prone) position. In Martin JT, Warner MA editors. Positioning and Anestesia in Surgery, ed 3. Philadelphia: WB Saunders, 1997, pp 155-195.

3. Cheng MA, Todorov A, Tempelhoff R, McHugh T, Crowder CM, Lauryssen $\mathrm{C}$. The effect of prone positioning on intraocular pressure in anesthetized patients. Anaesthesiology. 2001;95(6): 1351-1355.

4. Valdivia Uría, JG, Lanchares E, Villarroya S, Taberner Llop J, Abril Baquero G, Aranda Lasa JM. Nefrolitectomía percutánea: Técnica simplificada.(nota previa). Arch. Esp Urol. 1987,40(3): 177-180.

5. Valdivia Uria JG, Valle Gerhold JA, López López JA, Villarroya Rodriguez S, Ambroj Navarro M, Ramirez Fabián M, et al. Technique and complications of percutaneous nephroscopy: experience with 557 patients in the supine position. J Urol. 1998,160(6 Pt 1):1975-1978.
6. Valdivia-Uría JG, Valer J, Villarroya S, López JA, Bayo A, Lanchares $\mathrm{E}$, et al. Why is percutaneous nephroscopy still performed with the patient prone? J Endourol. 1990;4:350-359.

7. Fariña Pérez LA, Zungri Telo ER, Valdivia JG. La posición supina de Valdivia como mejor opción para la cirugía percutánea de los cálculos renales en el paciente con obesidad mórbida. Actas Urol Esp. 2005;29(10):997-1000.

8. Manohar MD, Prashant Jain MS, Mahesh Desai MS. Supine percutaneous nephrolithotomy: Effective approach to high-risk and morbidly patients. J Endourol 2007;21(1):44-49.

9. Shoma AM, Eraky I, El-Kenawy MR, El-Kenawy M, El-Ksppany H. Percutaneous nephrolithotomy in supine position: Technical aspects and functional outcome compared with the prone technique. Urology. 2002;60(3):388-392.

10. Ng MT, Sun WH, Cheng CW, Chan ES. Supine position is a safe and effective for percutaneous nephrolithotomy. $J$ Endourol 2004; 18(5):469-473.

11. Chedid Neto EA, Ibrahim Mitre A, Mendes Gómez C, Arap MA, Srougi M. Percutaneous nephrolitotomy with the patient in a modified supine position. J Urol. 2007,178(1):165-168.

12. Ibarlucea G, Scoffone C, Cracco C, Poggio M, Porpiglia F, Terrone $\mathrm{C}$, et al. Supine Valdivia and modified lithotomy position for simultaneous anterograde and retrograde endourological access. BJU Int. 2007,100(1):233-236.

13. Preminger DG, Assimos JE, Lingeman SY, Nakada MS, Pearle M, Wolf J. Chapter 1: AUA guideline on management of staghorn calculi: diagnosis and treatment recommendations. J Urol. 2005;173(6): 1991-1993.

14. El- Nahas AR, Shokeir AA, El-Assm AM. Colonic perforation during percutaneous nephrolithotomy: Study of risk factors. Urology. 2006,67(5):937-941.

Correspondencia autor: Dr. J.H. Amón Sesmero

Servicio de Urología. Hospital Rio Hortega.

Av. Cardenal Torquemada s/n - 47100 Valladolid. Tel.: 983420400

E-mail autor: amonsesmero@yahoo.es

Información del artículo: Original - Endourología

Trabajo recibido: octubre 2007

Trabajo aceptado: diciembre 2007 